\title{
Kennedy, Kroutchev et les missiles de Cuba Choix rationnel et responsabilité individuelle (Partie 1)
}

Jean-Yves Haine

\section{(2) OpenEdition}

Édition électronique

URL : http://journals.openedition.org/conflits/594

DOI : $10.4000 /$ conflits.594

ISSN : $1777-5345$

Éditeur :

CCLS - Centre d'études sur les conflits lilberté et sécurité, L'Harmattan

Édition imprimée

Date de publication : 1 mars 2000

ISBN : 2-7384-9520-6

ISSN : 1157-996X

\section{Référence électronique}

Jean-Yves Haine, « Kennedy, Kroutchev et les missiles de Cuba Choix rationnel et responsabilité individuelle (Partie 1) », Cultures \& Conflits [En ligne], 36 | hiver 1999 - printemps 2000, mis en ligne le 20 mars 2006, consulté le 30 mars 2021. URL : http://journals.openedition.org/conflits/594 ; DOI : https://doi.org/10.4000/conflits.594

Ce document a été généré automatiquement le 30 mars 2021.

Creative Commons License 


\title{
Kennedy, Kroutchev et les missiles de Cuba Choix rationnel et responsabilité individuelle (Partie 1)
}

\author{
Jean-Yves Haine
}

1 «The missiles crisis was a dramatic demonstration of human fallibility, of the degree to which political and military leaders are so often captives of misinformation, misjudgment, miscalculation. We all make mistakes. But if mistakes were to affect decisions relating nuclear forces, they will result in the destruction of nations ». Robert MacNamara $^{1}$ " I admit the human element seems to have failed us here... I don't think it is quite fair to condemn a whole program for a single slip-up ». Général Turgidson, Dr. Stangelove ${ }^{2}$

Dans l'histoire diplomatique de la guerre froide, la crise des missiles de Cuba restera sans aucun doute la plus grave, la plus intense et la plus dangereuse. Durant ces 13 jours fatidiques d'octobre 1962, Américains et Soviétiques se regardèrent droit dans les yeux, et l'humanité retint son souffle. Pour la première et la dernière fois, les deux supergrands se retrouvèrent directement face à face. Près de quarante ans après les faits, on éprouve quelques difficultés à saisir l'intensité de cette confrontation. Le discours du président Kennedy le 22 octobre, dévoilant la présence de missiles soviétiques à Cuba et annonçant la quarantaine autour de l'île, provoqua un choc sans précédent parmi la population occidentale, et déclencha sporadiquement des vagues de panique. ${ }^{3}$ Pour l'opinion publique, le spectre de la guerre nucléaire n'était plus une possibilité vague et lointaine, elle devint une probabilité proche et concrète. ${ }^{4}$ Parmi les officiels à Washington, et en particulier pour les responsables militaires, l'alarme fut bien réelle. Le Strategic Air Command (SAC) fut placé pour la seule fois de son existence en conditions de défense 2, ce qui signifiait, entre autres, la mise en alerte de 172 missiles, 1200 bombardiers nucléaires, 90 Atlas et 46 ICBM Titans. La puissance destructrice des forces du SAC dépassait les 6000 mégatonnes. ${ }^{5}$ Par ailleurs, une force d'invasion, la plus importante depuis la deuxième guerre mondiale, fut mise sur pied en Floride. Les plans d'attaque de Cuba comprenaient le déploiement d'une force terrestre 
de 150000 hommes, des milliers de sorties aériennes, la mobilisation de près de troisquarts des bateaux cargos de la côte Est, 42000 tonnes de matériels envoyés sur l'île, rien que pour la première semaine, $\mathrm{y}$ compris plus de 300 chars d'assaut $^{6}$. Les Soviétiques, pour leur part, placèrent leurs forces conventionnelles en alerte mais ne touchèrent pas au statut de leurs forces nucléaires. Par contre, les 42000 soldats soviétiques présents à Cuba se préparaient activement à une invasion américaine jugée imminente, et les 48 divisions cubaines étaient bien évidemment sur pied de guerre. Largement secrète ${ }^{7}$, cette mobilisation des arsenaux respectifs, y compris des moyens nucléaires, risquait de déboucher sur le bras de fer le plus meurtrier de l'histoire de l'humanité. L'opinion publique, qui ignorait la majorité de ces développements, se focalisa sur une joute bien plus symbolique lorsque Sorensen interpella Zorin à la tribune de l'ONU. Par sa portée, son ampleur, et son intensité, la crise de Cuba fut bien l'une des étapes décisives du XXème siècle. Dans l'imaginaire collectif, le déploiement des missiles à Cuba restera un événement qui faillit faire basculer le monde dans le cauchemar nucléaire, la seule confrontation où le sort de la planète dans sa globalité fut en jeu. Pour les principaux protagonistes, Kennedy et Kroutchev, la gravité de la crise et l'acuité du danger ne firent aucun doute. ${ }^{8}$ Tous deux devaient s'efforcer de garder un profil bas après la résolution de la crise, témoignage commun d'un soulagement partagé, mais aussi d'une culpabilité réciproque dans la dramatisation excessive de cette confrontation. ${ }^{9} \mathrm{Au}$-delà de ces aspects strictement militaires, cette confrontation fut principalement psychologique. Kroutchev, obnubilé par le statut, le prestige et le rang de l'Union soviétique, tenta de pallier cette infériorité stratégique par une gageure nucléaire. L'introduction dans le plus grand secret d'armes nucléaires à Cuba devait mettre les Etats-Unis devant le fait accompli et les obliger à reprendre les négociations sur l'Allemagne sur des bases paritaires nouvelles tout en préservant les acquis de la révolution cubaine. Le pari était essentiellement politique, autant sur la scène diplomatique, que sur le plan intérieur où les réformes ambitieuses de Kroutchev, notamment en matières agricoles, n'avaient guère atteint leurs objectifs. Face à ce défi, le président Kennedy, affaibli par le fiasco de la Baie des Cochons, restait déterminé à résoudre le problème récurrent de Cuba. Castro constituait en effet le talon d'Achille de l'équipe Kennedy, rassemblant l'élite intellectuelle et industrielle de la nation. Les intrigues répétées de la CIA, les multiples tentatives d'assassinats, les opérations parfois massives de déstabilisation donnaient à la Maison-Blanche une atmosphère de conspiration et de nervosité permanente. ${ }^{10} \mathrm{La}$ seconde épine du Président était un autre dossier, nettement plus important, le statut de Berlin. Depuis 1958, l'Union soviétique avait en effet multiplié les mises en garde et menacé à plusieurs reprises de signer un traité de paix avec l'Allemagne de l'Est. Le réarmement conventionnel et le redressement économique spectaculaire de la République Fédérale, qui coïncidait avec une détérioration accrue en République Démocratique, étaient perçus par Moscou comme une remise en cause du statut quo en Europe. Kroutchev tenta d'abord de résoudre ce problème en multipliant les ouvertures avec Eisenhower. Mais pour ce dernier, l'Europe était un tout et seules les armes nucléaires, de préférence européennes, permettaient de la défendre. La crédibilité de la dissuasion impliquait le maintien de l'engagement à Berlin. Kennedy pour sa part ne croyait pas aux représailles massives et refusait la nucléarisation de l'Allemagne. Conscient que le rapport des forces stratégiques était largement en faveur des Américains - le ratio est, en 1962, de 17 contre $1^{11}$ - il était inflexible sur Berlin mais savait que cette position privilégiée ne durerait pas. A l'automne 1962, Kennedy était prêt à tenter de trouver un 
arrangement raisonnable sur la question berlinoise, mais si Moscou se montrait intransigeant, peut-être fallait-il précipiter l'épreuve de force. Pour sa part, Kroutchev cultivait une sorte de complexe d'infériorité vis-à-vis du monde occidental, et se montrait parallèlement soucieux d'assurer le leadership du camp soviétique par rapport à son partenaire chinois. En bref, il était bien conscient de la précarité de sa position, mais avait besoin d'une victoire diplomatique. ${ }^{12} \mathrm{~A}$ Washington, on se préparait à une nouvelle confrontation sur Berlin et, dans cette mesure, le dossier cubain était intimement lié à la défense de l'Occident. A Moscou, les revendications et les ultimatums successifs du secrétaire général ne pouvaient pas rester longtemps lettre morte sans compromettre le rang et le prestige de l'Union soviétique. Pour Kroutchev, cette dimension essentiellement politique et psychologique de la rivalité entre l'Est et l'Ouest unissait les deux dossiers. De part et d'autre, on avait conscience que le moment de vérité approchait. La crise de Cuba combine donc à la fois une réalité stratégique, une composante nucléaire et une dimension psychologique. Cette triple caractéristique donne toute sa richesse à cet événement unique. Il n'est donc guère surprenant que cette crise demeure la plus étudiée par les historiens et les politologues de la guerre froide. ${ }^{13}$ Chaque décennie devait apporter son lot de révélations, de nouvelles interprétations, de débats houleux. Mais depuis la fin des années 1980 surtout, en partie sous l'impulsion de la perestroïka, de nombreux documents sont venus compléter l'état des connaissances. Plusieurs conférences se sont échelonnées à Moscou, La Havane, Cambridge, où des anciens officiels sont venus confier leurs témoignages. L'ouverture des archives aux Etats-Unis est aujourd'hui quasiment complète. Grâce aux enregistrements du président Kennedy durant les réunions de l'Exécutif Committee, une émanation ad hoc du National Security Council, rassemblant plus d'une vingtaine de conseillers, toutes les conversations entre responsables civils et militaires sont désormais disponibles. ${ }^{14}$ Malheureusement, il n'y a pas d'équivalent pour Moscou, où si les archives des affaires étrangères ont bien été en partie ouvertes, celles du Politburo en revanche demeurent encore largement classées. Néanmoins les chercheurs ont désormais cette opportunité remarquable de se mettre à la place des décideurs, d'évaluer en temps réel leurs décisions et leurs réactions. Cette exceptionnelle transparence dans un domaine particulièrement sensible fait de la crise de Cuba un cas d'école, aussi bien dans le domaine nucléaire que dans celui de l'étude de la décision. Elle permet en outre de prendre la juste mesure de l'impact des missiles de Cuba dans l'histoire de la guerre froide. A bien des égards, cette crise clôt en effet le premier chapitre de la confrontation Est-Ouest et ouvre une période plus pacifique dont la détente sera le point culminant. ${ }^{15}$ La position défendue dans cet article est double. Il s'agit tout d'abord de rechercher et de prendre au sérieux les intentions des acteurs. Cette constatation de bon sens est paradoxalement absente en relations internationales dont les courants dominants tendent à ne considérer les préférences des acteurs que de manière marginale. ${ }^{16}$ Le plus souvent formulées en termes de contraintes, les analyses classiques traditionnelles estiment que l'étude des intentions est trop réductrice et aléatoire pour comprendre le système international. Cette marginalisation est regrettable à plusieurs titres : tout d'abord, les généralisations liées aux contraintes inhérentes au système international négligent l'autonomie des décideurs et adoptent une conception déterministe de l'histoire. C'est dénaturer la politique internationale que d'oublier que celle-ci est fondamentalement une succession de choix complexes, et opérés le plus souvent dans la douleur, d'alternatives suggérées mais évincées, d'options laborieusement définies mais finalement rejetées. 
Ensuite, cet ordre de succession revêt une importance capitale. Les préférences des acteurs sont situées historiquement, l'étude minutieuse de leurs sources et de leurs origines demeure impérative pour reconstituer précisément l'enchaînement des causes et des effets sans tomber dans le piège de l'anachronisme. l'histoire est vécue prospectivement mais étudiée rétrospectivement. Comme le notait D. Acheson en exergue à ses mémoires, «History is lived forwards but it is written in retrospect. We know the end before we consider the beginning and we can never wholly recapture what it was like to know the beginning only ». Acheson Dean, Present at the creation. My years in the State Department, W.W. Norton, 1969, p. i. ]] Ces préférences ne se forment pas ex nihilo, pour une large part, elles sont le fruit d'expériences passées, de leçons apprises, de mémoires sélectives, et de trajectoires personnelles et collectives particulières. Enfin, l'analyse des intentions est une étape nécessaire pour identifier les responsabilités et pour en mesurer le poids et l'impact sur le décideur. Dans le domaine nucléaire, ces responsabilités sont considérables. Sans l'étude des intentions, il ne peut $\mathrm{y}$ avoir d'éthique de responsabilité. Le second argument vise à démontrer l'importance de la rationalité limitée en relations internationales. Une étude récente sur la fin de la guerre froide articulait quatre éléments sans lesquels cet épisode important ne peut être correctement interpreté : «Four basic and related problems confront the decision maker and the scholar : the uncertainty under which decision makers must frequently operate, which can translate into an ambiguous historical record for scholars; the immeasurability of the variables that both decision makers and political scientists think are important; the complexity of the situation that decision makers face and scholars seek to understand; and the incomparability of these situations over time and across cases ». Ces caractéristiques se retrouvent dans toute étude de la décision. Voir Wohlforth William C. (Ed.), Witnesses to the End of the Cold War, The John Hopkins University Press, 1996, pp. 261 et suiv. ]] Les simplifications, inhérentes au modèle de la rationalité substantielle, et les formalisations qu'impliquent les objectivations des critères de choix, sont tellement éloignées de la réalité complexe et incertaine, telle qu'elle est perçue et vécue par les décideurs, qu'elles ne nous apprennent rien de très utile sur les mécanismes liés à la prise de décision. Nier la complexité et négliger l'incertitude, c'est dresser des schémas abstraits, arbitraires et normatifs à partir desquels sont évalués des comportements qualifiés d'irrationnels lorsqu'ils s'écartent des modèles proposés. Cet article part d'une prémisse opposée. Il entend prendre pleinement en compte les effets de cette complexité et les impacts de cette incertitude sur les processus de décision. Car avant de produire des logiques bureaucratiques et politiques, la rationalité limitée entraîne d'importantes conséquences sur les perceptions, les raisonnements et les décisions des acteurs. Parce que les décideurs doivent rendre cette complexité intelligible et parce que les signaux qu'ils reçoivent, et qu'ils émettent, sont le plus souvent ambigus, les processus cognitifs liés à l'incertitude, nous permettent de mieux comprendre pourquoi les actes posés sur la scène internationale reçoivent des interprétations si divergentes et pourquoi les responsables politiques sont si soucieux de leur image, de leur réputation, et de leur crédibilité. Associée généralement aux processus organisationnels, cette rationalité limitée, qui favorise la forme sur le fond ${ }^{17}$, et privilégie les incréments successifs au détriment des changements spectaculaires, doit se décliner en priorité au niveau des responsables eux-mêmes. Face aux effets de la complexité, l'analyste doit adopter pour sa part une démarche modeste parce que les interprétations sont le plus souvent multiples et parfois contradictoires, mais minutieuse parce que les détails ont toujours leur 
importance. En un mot, cet article défend une conception humaniste de la politique internationale, qui replace les individus au cœur des processus décisionnels. Il propose une méthodologie de l'étude de la décision basée davantage sur l'immersion dans les détails d'un seul événement que sur des généralisations à partir d'une multiplicité de cas. ${ }^{18}$ Sa première partie sera consacrée à une lecture critique du modèle rationnel de la décision, tel qu'il est développé dans la nouvelle édition d'Essence of Decision, et reproduit dans ce numéro. La seconde présentera une approche cognitive du processus de décision, en insistant sur les conséquences de la rationalité limitée pour les responsables politiques. La troisième fera le point sur l'historiographie de la crise de Cuba et tentera succinctement de mettre en lumière les éléments fondamentaux qui permettent d'expliquer les origines de cette crise et les sources de sa résolution. Avant d'aborder plus en détail le choix rationnel, une brève présentation des deux modèles alternatifs, est cependant nécessaire. Les trois modèles de décision proposés par Graham Allison dans son ouvrage de 1971 sont désormais bien connus de tous les chercheurs et étudiants de science politique en général et des relations internationales en particulier. Il n'est pas cependant inutile de rappeler que l'ambition de ces trois paradigmes demeure modeste. Il s'agit en effet de présenter des grilles de lectures permettant à tout un chacun de mieux décrypter les informations qu'il reçoit quotidiennement en abondance. En prenant en compte les contraintes de temps - le lecteur d'un grand quotidien ou le téléspectateur des informations télévisées doit assimiler une grande quantité d'informations en très peu de temps - ces modèles d'explications apportent des réponses simples à ceux qui tentent de rechercher et d'expliquer la cause d'un événement. Pourquoi Boris Eltsine a-t-il démissionné le 31 décembre 1999? Pourquoi l'Alliance atlantique a-t-elle mené une campagne aérienne au Kosovo ? Pourquoi les Etats-Unis maintiennent-ils un embargo contre Cuba? Les trois modèles, rationnel, organisationnel et gouvernemental, qui rendent explicite ce qui le plus souvent est implicite dans le raisonnement explicatif, renvoient à des logiques différentes : celle de l'intention, celle du phénomène bureaucratique, et celle du processus politique.

\section{NOTES}

1. Cité par Blight James et Welch David A., «Risking 'The Destruction of Nations' : Lessons of the Cuban Missile Crisis for New and Aspiring Nuclear States », Security Studies, summer 1995, vol. 4, n4, p. 811.

2. Cité dans Henriksen Margot A. Dr. Strangelove's America, Society and Culture in the Atomic Age, University of California Press, 1997, pp. 321-322.

3. « A considerable number of people from London to Tokyo thought they might not live to see another dawn. Young people in particular became deeply alarmed. In Washington, shovels and sandbags were sold out at hardware stores. In some cities, food hoarding panics stripped supermarkets bare... The public was calm only in Moscow, where the press did not mention until after the crisis was over that the 
squabble had something to do with nuclear missiles ». Voir Weart Spencer R., Nuclear Fear : A History of Images, Harvard University Press, 1988, p. 259.

4. Un sondage Gallup réalisé le lendemain montre que pour plus d'un Américain sur cinq, le blocus allait déclencher la troisième guerre mondiale. Cité dans White Mark J., Missiles in Cuba : Kennedy, Khrushchev, Castro and the 1962 Crisis, Ivan R. Dee, 1998, p. 112.

5. Pour le détail des forces en alerte, voir Sagan Scott D., « Nuclear Alerts and Crisis Management », International Security, spring 1985, vol. 9, n4, pp. 99-139.

6. Sur les plans successifs d'invasion, voir Desch Michael, «'That Deep Mud in Cuba' : The Strategic Threat and U.S. Planning for a Conventional Response during the Missile Crisis ", Security Studies, winter 1991, vol. 1, n², pp. 317-351. L'auteur estime que les plans étaient bien plus flexibles que ne le pensaient les responsables de la MaisonBlanche.

7. L'ordre de passer en Def. Con 2 fut transmis en clair par le général Thomas Power, commandant-en-chef du SAC. Sur cet incident, ignoré par les membres de l'Ex-Comm, voir Garthoff Raymond L., Reflections on the Cuban Missiles Crisis, The Brookings Institution, 1989, p. 62.

8. Fin octobre, Kennedy confia aux leaders du Congrès que les Etats-Unis avaient résolu l'une des «plus graves crises de l'humanité ». Cité par Beschloss Michael R., The Crisis Years, Kennedy and Khrushchev 1960-1963, Edward Burlingame Books, 1991, p. 545. Kroutchev pour sa part déclara au Soviet Suprême, en novembre 1962, que le monde avait été « tout près de l'abysse ». Cité par Winkler Allan M., Life Under a Cloud, American Anxiety about the Atom, Oxford University Press, 1993, p. 179. Le britannique McMillan devait reconnaître que cette crise était bien « la plus dangereuse que le monde avait connu depuis la fin de la deuxième guerre mondiale ». Voir son introduction dans Kennedy Robert F., Thirteen Days, A Memoir of the Cuban Missile Crisis, New American Library, 1969, p. 17.

9. En partie pour ne pas humilier davantage l'Union soviétique, Kennedy demanda à la presse américaine d'éviter tout triomphalisme déplacé. Voir Reeves Richard, President Kennedy : Profile of Power, Simon \& Schuster, 1993, p. 424.

10. Parmi ces opérations de déstabilisation, la plus importante était l'opération Mongoose, qui faisait l'objet d"une âpre bataille bureaucratique entre le State Department, la CIA et Robert Kennedy. Le Président pour sa part refusait l'implication directe des troupes américaines, ce qui compromettait la finalité et l'efficacité de ces opérations. Sur ces questions, on se reportera avec précaution à Hersh Seymour, The Dark Side of the Camelot, Little, Brown, 1997 ; May Ernest R. et Philip Zelikow, "Camelot Confidential », Diplomatic History, Fall 1998, vol. 22, n4, pp. 642-661. Sur la connaissance par les Soviétiques de ces opérations, voir Fursenko Aleksander et Naftali Timothy, One Hell of a Gamble, Khrushchev, Castro \& Kennedy, 1958-1964, W.W. Norton \& Company, 1997, pp. 132-166.

11. Cette supériorité américaine rendait Kroutchev furieux. Ce rapport de force défavorable, nous le verrons, joua un rôle ambigu dans la décision d'envoyer des missiles nucléaires à Cuba. Sur le ratio, voir Gribkov Anatoli I. et Smith William Y., Operation Anadyr : U.S. and Soviet Generals Recount the Cuban Missiles Crisis, Edition q, 1994, pp. 10-11.

12. Sur les différentes étapes des crises de Berlin du côté américain, Trachtenberg Marc, History and Strategy, Princeton University Press, 1991, Chap. 5, pp. 169-234 ; Trachtenberg Marc, A Constructed Peace, The Making of the European Settlement 
1945-1963, Princeton University Press, 1999, Chap. 7 et 8, pp. 251-351 ; Schwartz Thomas A., " The Berlin Crisis and the Cold War ", Diplomatic History, winter 1997, vol. 21, n¹, pp. 139-148. Du côté soviétique, Talbott Strobbe (Ed.), Khrushchev Remembers, The Last Testament, Bantham Books, 1974 ; Zubok Vladislav et Constantine Pleshakov, Inside the Kremlin's Cold War, From Stalin to Khrushchev, Harvard University Press, 1996, Chap. 7 et 8, pp. 210-274 ; Zubok Vladislav, "Khrushchev and the Berlin Crisis ", Cold War International History Project Working Paper, n6, may 1993.

13. Certains auteurs estiment cependant que la crise de Cuba est bien trop exceptionnelle pour en retirer des leçons pertinentes à d'autres cas. "We must ask ourselves whether the uniqueness of the crisis does not destroy its value as an archetype, or worse, make it a profoundly misleading subject for reflection ". Cohen Eliot A., "Why We Should Stop Studying the Cuban Missiles Crisis », The National Interest, winter $1986, \mathrm{n}^{\circ} 2$, p. 5 . A cette question, ma réponse est négative. Plus on fouille dans les archives, plus on en apprend sur les processus de décision. La crise de Cuba ne déroge pas à cette constatation.

14. Ces conversations, dont certaines sont disponibles depuis les années 1980, sont retranscrites dans Zelikow Phillip et Ernest May, The Kennedy Tapes, Inside the White House During the Cuban Missile Crisis, Belknap Press of Harvard University, 1997. La mauvaise qualité des bandes sonores a rendu la tâche particulièrement difficile. Pour se rendre compte de l'étendue du travail, il est désormais possible d'écouter une partie de ces conversations sur le site web du National Security Archives, http://www. nsa.com. 15. Ce qui fera dire à Thomas Schelling : "The Cuban missile crisis was the best thing to happen to us since the Second World War. It helped us avoid further confrontations with the Soviets ; it resolved the Berlin issue ; and it established new basic understandings about U.S.-Soviet interaction ». Pour sa part, Sergo Mikoyan reconnaît que « if there had been no Cuban missile crisis, we should perhaps have organized it ». Schelling et Mikoyan sont cités dans Lebow Richard Ned, « The Traditional and Revisionist Interpretations Reevaluated : Why Was Cuba a Crisis ? ", in Nathan James A. (Ed.), The Cuban Missile Crisis Revisited, St. Martin's Press, 1992, pp. 178-181.

16. Comme le note A. Moravcsik, « the liberal assumption - in particular that what states want is the primary determinant of what they do - , may seem commonsensical, even tautological. Yet mainstraim IR theory has uniformly rejected such claims for the past half-century. At the heart of the two leading contemporary IR theories, realism and institutionalism, is the belief that state behavior has ironic consequences ». Voir Moravcsik Andrew, « Taking Preferences Seriously : A Liberal Theory of International Politics », International Organization, autumn 1997, vol. 51, n 4, p. 521-522.

17. Pour cette raison, $H$. Simon, qui fut le premier à proposer ce modèle, préfère utiliser le terme de rationalité « procédurale ». Voir Simon Herbert, Models of Bounded Rationality, MIT Press, 1982.

18. Comme le précise bien l'adage anglais, « As always, devil lies in detail ». 
INDEX

Index géographique : Caraibes, Etats-Unis, Russie

Mots-clés : professionels de la politique, Relations Internationales, Stratégie

Index chronologique : guerre froide 